\title{
Revendications musulmanes sur la gouvernance de la laïcité au Burkina Faso
}

Yacouba Ouédraogo

\section{OpenEdition}

12 Journals

Édition électronique

URL : http://journals.openedition.org/anthropodev/691

DOI : 10.4000/anthropodev.691

ISSN : 2553-1719

Éditeur

APAD - Association pour l'anthropologie du changement social et du développement

Édition imprimée

Date de publication : 1 décembre 2018

Pagination : 119-140

ISBN : 979-10-93476-06-3

ISSN : 2276-2019

Référence électronique

Yacouba Ouédraogo, « Revendications musulmanes sur la gouvernance de la laïcité au Burkina Faso », Anthropologie \& développement [En ligne], 48-49 | 2018, mis en ligne le 18 juillet 2019, consulté le 19 août 2019. URL : http://journals.openedition.org/anthropodev/691 ; DOI : 10.4000/ anthropodev.691

La revue Anthropologie \& développement est mise à disposition selon les termes de la Licence Creative Commons Attribution 4.0 International. 


\title{
Revendications musulmanes sur la gouvernance de la laïcité au Burkina Faso
}

\author{
Yacouba Ouédraogo
}

Le présent article entend proposer une analyse des processus ayant conduit à la mise à l'agenda de la problématique de la gouvernance de la laïcité au Burkina Faso. Dans un contexte de réislamisation en Afrique subsaharienne, il essaie de donner un regard sur l'émergence de l'islam dans l'espace public dans ce pays où le christianisme joue un rôle important. Au Burkina Faso, les associations musulmanes francophones, non satisfaites de la gestion de la laïcité par l'État, engagent une lutte à partir du début des années 1990, non pas pour remettre en cause la laïcité constitutionnelle, mais pour réclamer plus d'équité dans le traitement des confessions religieuses. Elles demandent l'institution d'un observatoire de la laïcité. La question émerge dans l'espace politique à la faveur des réformes institutionnelles entreprises par l'État en 2011. Le forum national sur la laïcité, organisé en 2012, a été un moment fort de débats, qui traduit la légitimation du sujet et qui révèle les différentes conceptions de la laïcité qui traversent la société burkinabè. Les revendications musulmanes ont conduit à une coproduction d'action publique certes, mais elles ont aussi confirmé le développement d'un renouveau islamique et l'émergence d'une société civile musulmane au Burkina Faso.

This article intends to propose an analysis of the processes that led to the scheduling of the issue of the governance of secularism in Burkina Faso. In a context of an Islamic revival in sub-Saharan Africa, it tries to give a look at the emergence of Islam in the public space in this country where Christianity plays an important part. In Burkina Faso, French speaking Muslim associations, unsatisfied with the management of secularism by the State, engaged a struggle in the early 1990s, not to question constitutional secularism, but to ask for more equity in the treatment of Religious denominations. They call for the establishment of an observatory of secularism. The issue emerges in the political sphere through the institutional reforms undertaken by the State in 2011. The national forum on secularism organized in 2012 was an important moment of debates that 
expresses the legitimation of the topic and that reveals the different conceptions on secularism in the Burkinabè society. Not only Muslim claims lead to a co-production of public action, but it also confirmed the development of an Islamic revival and the emergence of a Muslim civil society in Burkina Faso.

\section{Introduction}

Depuis plusieurs décennies, l'islam marque son émergence dans l'espace public en Afrique subsaharienne sous la forme d'un renouveau islamique ou d'un mouvement de réislamisation (Holder et Saint-Lary, 2014). Dans cette dynamique, l'un des sujets portés dans le cadre du débat public par le champ islamique reste celui la laïcité (Holder et Sow, 2013), disposition juridique adoptée par la plupart des anciennes colonies d'Afrique noire. Les fondements de cette laïcité à la française que sont l'égalité de traitement entre les religions, la liberté de culte et la séparation institutionnelle entre l'État et la religion sont diversement réappropriés dans les pays d'Afrique de l'Ouest (Kaag et Saint-Lary, 2011). Au Burkina Faso (ex Haute-Volta), ce principe normatif de laïcité est laconiquement repris dans les textes constitutionnels des $\mathrm{I}^{\mathrm{re}}, \mathrm{II}^{\mathrm{e}}$, $\mathrm{II}^{\mathrm{e}}$ et $\mathrm{IV}^{\mathrm{e}}$ Républiques adoptés depuis $1960^{1}$. À l'instar de la plupart des anciennes colonies françaises d'Afrique occidentale, la mention de ce principe dans les textes ne signifie pas pour autant l'existence d'un corpus élaboré (Traoré, 2010 ; Vitale, 2013; Ouédraogo, 2013). Toutefois, plusieurs dispositions de la constitution burkinabè du 11 juin 1991 rappellent les principes fondamentaux de la laïcité : la liberté de conscience, la liberté de croyance et la neutralité de l'État. Le concept de laïcité du fait de son extranéité est perçu au Burkina Faso comme un produit d'importation (Vitale, 2013 : 303304). En l'absence d'une définition juridique de la laïcité et du fait des enjeux observés au cours de l'évolution du pays, les différentes confessions religieuses notamment les musulmans et les chrétiens ont produit des lectures et des interprétations des faits parfois différenciées. Dans la Haute-Volta nouvellement indépendante, l'hégémonie chrétienne est manifeste dans la vie politique et sociale (Guy, 1981 : 231-232 ; Otayek,

\footnotetext{
${ }^{1}$ Novembre 1960, juin 1970, novembre 1977 et juin 1991.
} 
1984: 301). Malgré son poids démographique minoritaire, l'action sociopolitique de l'Église et sa visibilité ont fait d'elle une communauté qu'on peut qualifier de "minorité dominante " (Bouron, $2010: 80$ ). Dans un rapport ${ }^{2}$, les responsables de la Communauté musulmane n'ont pas manqué de rappeler que, sous la lre République, le président Maurice Yaméogo, ancien élève d'un séminaire catholique, se disait " une créature de l’Église » et considérait la Haute-Volta comme la « fille chérie de l'Église en Afrique " (Deniel, 1970 : 325). Cette considération de la puissance de l'Église catholique s'estompe quelque peu avec l'arrivée au pouvoir, en 1966, d'Aboubacar Sangoulé Lamizana, président de confession musulmane. Avec l'ouverture de la diplomatie voltaïque vers le monde arabe, l'introduction de l'enseignement de la langue arabe dans le cycle secondaire public, la rupture des relations diplomatiques entre la HauteVolta et Israël en 1973, l'adhésion de la Haute-Volta à l'Organisation de la conférence islamique (OCI) en 1975, l'Église catholique a tenu à son tour un discours critique vis-à-vis de l'État. En effet, certains responsables de cette Église ont émis des inquiétudes quant à la coloration islamique que prendrait l'État voltaïque (Audouin et Deniel, 1975: 70 ; Otayek, 1997 : 236), tandis que d'autres par une lettre pastorale du 23 avril 1978 lue dans les églises de Ouagadougou ont dénoncé une atteinte à la laïcité (Otayek, 1997: 236-237). Après la chute du régime constitutionnel de Sangoulé Lamizana à la suite d'un coup d'État en novembre 1980, quatre régimes d'exception ont gouverné le pays entre 1980 et 1990 . Vis-à-vis de l'État, les différentes confessions religieuses observent un certain mutisme durant cette parenthèse de régimes militaires et notamment sous le Conseil national de la révolution de Thomas Sankara (Otayek, 1997: 238; Ouédraogo, $2013: 12$ ).

C'est avec le retour du processus démocratique en 1990 que le débat sur la laïcité refait surface. Le sujet est relancé par des organisations musulmanes qui, si elles ne remettent pas en cause la laïcité comme principe normatif dans la société burkinabè multiconfessionnelle, ne semblent pas satisfaites de la gestion de la laïcité par l'État. Elles réclament

\footnotetext{
2 II a été écrit par des membres dirigeants de la Communauté musulmane de Haute-Volta en réponse à un questionnaire qui leur a été soumis dans le cadre d'une étude.
} 
une meilleure gouvernance ${ }^{3}$ de la laïcité, bien que celle-ci ne soit pas jusque-là définie. Ce cas de figure est d'ailleurs observable dans d'autres pays d'Afrique subsaharienne comme en Côte d'Ivoire où les musulmans ont réclamé un traitement égalitaire des confessions religieuses (MiranGuyon, 2013 : 324). Ces débats sur la laïcité sont à inscrire dans une dynamique plus large à l'échelle des pays francophones d'Afrique où l'on observe le développement d'un renouveau islamique (Holder et Saint-Lary, 2014) et l'émergence d'une "société civile musulmane " (Holder et Sow, 2013 : 25). Celle-ci s'appuie sur le cadre démocratique ambiant pour ainsi poser le problème de la gestion de la laïcité " par le bas " (Holder et Sow, 2013 : 25). Au sein de la diversité musulmane burkinabè, ce débat est surtout porté et animé par des structures qui regroupent spécifiquement des lettrés musulmans formés dans le système éducatif officiel dont la langue d'enseignement est le français (Traoré, 2010 : 42 ; Ouédraogo, 2013). D'autres courants ont des perceptions plus distanciées par rapport à la laïcité, à l'image des wahhabites et de certains arabisants pour qui la laïcité est un phénomène étranger à l'islam et par conséquent antiislamique (Traoré, $2010: 32$; Ouédraogo, 2013 : 20-21). Ce qui va justifier l'usage de la mention "francophone " pour désigner précisément cette catégorie de musulmans ou leurs groupes ou encore leurs cadres d'expression ${ }^{4}$. Les démarches de ces musulmans francophones ont dû aussi être influencées par les problématiques discutées au sein des réseaux islamiques francophones à l'image du Colloque international des musulmans de l'espace francophone (CIMEF). La première édition de ce colloque s'est tenue en Côte d'Ivoire en 2000 sous la direction de Tariq Ramadan (islamologue suisse) et la quatrième à Ouagadougou en 2006 (Holder et Sow, 2013 : 25-26). Lors du premier colloque, les participants ont dressé l'état des lieux de la laïcité dans les pays de l'espace francophone et ont discuté de sa terminologie (CIMEF, 2001). L'intérêt du

\footnotetext{
${ }^{3}$ Nous avons choisi d'employer le terme gouvernance au détriment du terme gestion plus usité par les acteurs. C'est un concept qui a acquis un droit de cité dans les discours des intellectuels africains depuis sa vulgarisation par les grandes institutions internationales à travers la promotion de la bonne gouvernance.

4 II sera ainsi question dans cet article de musulmans francophones, d'intellectuels musulmans francophones, d'élite musulmane francophone, de mouvement musulman francophone, etc.
} 
cas burkinabè est que la démarche des musulmans sous forme de plaintes et de revendications a abouti à l'organisation en 2012, par l'État, d'un forum national sur la laïcité. Cet exemple nous semble pertinent pour analyser les processus de formulation et de publicisation de problèmes par des acteurs sociaux, et les conditions dans lesquelles ils sont mis à l'agenda politique. L'objectif est de montrer comment les revendications musulmanes autour de la question de la laïcité ont pris forme et comment elles ont conduit à un débat public à travers l'organisation par l'État du forum national.

Il s'agit de chercher à comprendre les facteurs de mise à l'agenda, les ressources et les stratégies développées par les différents acteurs (État, partis politiques et communautés religieuses et coutumières) pour traduire le problème, les logiques de conflits et de coopération enregistrées, ainsi que les modalités de mise à l'agenda. Les résultats que nous présentons sont notamment issus d'une exploitation combinée des archives des rapports détaillés des assises du Conseil consultatif sur les réformes politiques (CCRP) et du forum de la laïcité, des coupures de presse, des discours et des entretiens avec des musulmans (responsables et simples fidèles). La présentation s'articule en deux points; le premier montre la phase de mobilisation collective musulmane autour de la formulation du problème de la laïcité et le deuxième analyse la phase de mise à l'agenda avec la décision d'organiser le forum, et les débats qui l'ont traversé.

Mobilisations et dénonciations musulmanes sur les pratiques de l'État en termes de laïcité

La fin des années 1980 coïncide avec l'émergence de mouvements intellectuels francophones musulmans. Il s'agit notamment de l'Association des élèves et étudiants musulmans au Burkina (AEEMB) et du Cercle d'études, de recherche et de formation islamique (CERFI) fondés respectivement en 1986 et 1989 . Jusqu'à cette période, les lettrés musulmans francophones militaient dans les trois associations créées depuis l'indépendance: la Communauté musulmane de Haute-Volta en 1962, le Mouvement sunnite de Haute-Volta en 1973 et l'Association 
islamique de la Tidjania de Haute-Volta en $1979^{5}$. Désormais, il existe deux associations islamiques spécifiquement francophones. L'AEEMB s'est fixé pour objectif de promouvoir l'islam dans le milieu scolaire et estudiantin tandis que le CERFI entend réaliser le même objectif dans le milieu de l'administration publique et privée. Le CERFI compte d'ailleurs parmi ses membres fondateurs des anciens militants de l'AEEMB devenus fonctionnaires. Ces deux associations du fait du cursus scolaire et universitaire de leurs membres utilisent couramment la langue française. $\mathrm{Ne}$ voulant pas être en marge du développement socio-économique du pays entrepris par l'État, le courant musulman francophone entend participer à la réalisation des projets étatiques aux côtés des autres confessions religieuses. Ainsi, son discours s'accommode de la laïcité de l'État et de la diversité religieuse sur le terrain. Mais, la préoccupation des associations d'intellectuels musulmans francophones porte plus sur le traitement équitable des confessions religieuses. Ce courant musulman va construire tout un discours et un argumentaire autour de la gestion de la laïcité au gré des événements.

\section{Les interpellations ponctuelles}

Les interpellations ponctuelles sont des réactions par rapport à des faits ou événements que des musulmans estiment mettre la laïcité en péril. En effet, en 1991, une lettre anonyme adressée au ministre de l'Information et de la Culture et dont les auteurs se disent être des musulmans affiliés aux trois grandes associations islamiques du Burkina met en garde l'État et les chrétiens contre les inégalités de traitement constatées dans la gestion des confessions religieuses en ces termes :

"Nous venons par cette mise en garde, vous entretenir d'un sujet qui nous tourmente depuis des années. La goutte d'eau fait déborder le vase dit-on et c'est la raison pour laquelle nous pensons qu'il est temps de vous sensibiliser et de vous mettre en garde... Mardi 25 décembre 1990. Fête de la nativité... Le ministre décide:

\footnotetext{
5 La Communauté musulmane de Haute-Volta, structure la plus ancienne, regroupait l'ensemble des courants islamiques avant l'émancipation de certaines tendances dont le Mouvement sunnite de Haute-Volta d'obédience wahhabite et l'Association de la Tidjania de Haute-Volta d'inspiration confrérique.
} 
les mass média, patrimoine de tous les Burkinabè sont à la disposition des chrétiens... la télévision qui d'ordinaire n'émet que les seuls samedis et dimanches dans la journée s'organise et dès $12 \mathrm{~h} 30$, elle émet et diffuse le message chrétien jusqu'à la nuit... Mardi 16 avril 1991. Fête de fin du jeûne musulman. Journée chômée et payée... la télévision reste muette, plus muette qu'une carpe dans la journée. Cette fête musulmane n'a pas de signification... On peut continuer la série, mais nous vous faisons grâce de la suite ${ }^{6} »$.

On retrouve, à travers ce passage, un condensé des frustrations ressenties par l'élite musulmane francophone. En outre, en 1992, les responsables de la Communauté musulmane du Burkina Faso $(\mathrm{CMBF})^{7}$ ont eu une audience avec le ministre d'État Roch Marc Christian Kaboré. La délégation était conduite par Toumani Triandé à l'époque président de la CMBF. Le premier sujet inscrit à l'ordre du jour par les responsables musulmans portait sur le non-respect de laïcité par l'État lui-même ${ }^{8}$. Si l'on ignore l'aspect sur lequel portait cette plainte, l'on peut en revanche se demander s'il n'y aurait pas un lien entre la lettre anonyme et la démarche des responsables musulmans en 1992. La raison en serait qu'avec le démarrage du processus démocratique et la tenue effective des élections présidentielles en novembre 1991, une certaine libéralisation de la vie sociopolitique était amorcée. Ailleurs, la religion musulmane a été dynamisée dans les établissements publics d'enseignement grâce à l'action éducative de l'AEEMB (Traoré, 2010: 35). Cela a suscité de nombreux problèmes dont la question du port du voile islamique en milieu scolaire. Les responsables de l'AEEMB ont dû saisir à plusieurs reprises les autorités du ministère des Enseignements secondaire et supérieur pour évoquer la question sous forme de plainte (le non-respect du principe de liberté religieuse). Les plaintes et interpellations formulées à partir des années 1990 ont poussé le ministère à faire sortir plus tard dans les années 2000 une circulaire pour résoudre le problème. Le texte précise que l'interdiction du voile islamique ne repose pas sur un texte légal et par conséquent invite les acteurs concernés à plus de retenue pour ne pas

\footnotetext{
${ }^{6}$ Archives de la présidence du Faso, Centre des archives nationales du Burkina (7V 485).

${ }^{7}$ La délégation comprend une majorité de cadres intellectuels francophones.

${ }^{8}$ Document $n^{\circ}$ 018/CMBF/92 du 9 mars 1992 (Archives nationales du Burkina, 7V 485).
} 
dégrader le climat social ${ }^{9}$. La presse islamique naissante fut également une tribune où le problème de la laïcité a été parfois évoqué.

\section{La presse islamique, tribune interne de débat sur la laïcité}

Les quelques organes de presse musulmans ${ }^{10}$ nés dans la mouvance de la libéralisation de la vie sociopolitique des années 1990 ont aussi servi d'espace à la construction d'un discours sur la laïcité au Burkina Faso. À l'analyse des différents écrits, les argumentaires se construisent autour de deux thématiques. Le premier argumentaire rejoint les revendications en Côte d'Ivoire (Miran-Guyon, 2013) et condamne le fait d'une laïcité " deux poids deux mesures ". Al-Maidane, bimensuel islamique animé par des cadres francophones, rappelle en 1999 trois principes fondamentaux de la laïcité que sont la liberté de conscience, la liberté de culte et la noningérence de l'État dans les affaires religieuses et s'interroge sur l'effectivité du caractère laïc de l'État burkinabè. L'attitude de l'État est critiquée pour ce qui concerne le troisième principe. Dans le réquisitoire des faits dressés, le journal dénonce la participation de la mairie de Ouagadougou à la fête de Noël par des distributions de cadeaux aux enfants. Par un procédé comparatif, il renchérit en soulignant le traitement inégalitaire des fêtes chrétiennes et musulmanes :

"Pas plus longtemps que le 28 mars dernier, les musulmans de notre pays ont célébré la fête de Tabaski. Le lendemain lundi, le travail a repris dans les administrations publiques. Une semaine après, c'était autour des chrétiens de fêter la Pâque. Le lendemain lundi de Pâques était d'office chômé et payé sur toute l'étendue du territoire burkinabè. Sans aiguiser la haine et la rancœur entre les confessions religieuses, nous pensons que les musulmans ont droit également à leur lundi de Tabaski ou leur lundi de Ramadan ${ }^{11}$ ".

\footnotetext{
${ }^{9}$ Circulaire $n^{\circ}$ 009/MESSRS/SG du 25 janvier 2006.

10 L'Appel créé en 1994 est animé par des intellectuels de l'AEEMB et du CERFI, An Nasr fondé en 1997 est un journal de l'AEEMB et Le Cerfiste fondé en 2006 est l'organe de presse du CERFI. Al-Maidane et La Preuve respectivement créés en 1999 et en 2007 sont animés aussi bien par des membres de l'AEEMB que du CERFI.

${ }^{11}$ Al-Maidane, bimensuel islamique, $n^{\circ} 3$ du 15 au 31 mai 1999, p. 3.
} 
En 2000, dans le cadre d'une relecture de la loi portant sur les fêtes légales, un projet de loi soumis et débattu à l'Assemblée nationale a pris en compte ce problème de "lundi de Pâques ". La loi adoptée mentionne que le lendemain d'une fête légale est désormais chômé et payé si ladite fête tombe un dimanche ${ }^{12}$.

Le deuxième argumentaire essaie de donner des orientations en dégageant les contours de la laïcité selon la perception de certains musulmans. II réfute l'adoption d'une laïcité importée, une sorte de laïcité exotique. C'est en substance ce qui ressort dans ce point de vue :

"Pour qu'un État ou un gouvernement ne soit pas mis à quia, il doit vivre sa laïcité et non la laïcité des autres... II ne sert à rien de voter des lois qui ne seront ni respectées, ni suivies. Si nul n'est censé ignorer la loi, la loi quant à elle est aussi censée ne pas ignorer la société et ses disparités ${ }^{13} \gg$.

Un autre point de vue abonde dans ce sens en soulignant la nécessité d'une laïcité endogène :

"La laïcité est inscrite d'office dans certaines constitutions au mépris des règles de procédure de consultation populaire. C'est le cas des constitutions octroyées, souvent en désaccord avec la réalité sociale des pays concernés... C'est le peuple qui produit la constitution et la loi et non l'inverse ${ }^{14}{ }^{\prime \prime}$.

Ces points de vue d'une frange des musulmans ne donnent pas une définition précise de la laïcité même s'ils en dessinent les contours. Toutefois, ils estiment que la laïcité si elle doit être appliquée dans le contexte africain, doit être définie sur la base des réalités culturelles locales. Autrement dit, une éventuelle laïcité à adopter au Burkina Faso devrait tenir compte de la diversité religieuse et du poids sociodémographique de chaque religion dans l'espace public. Hormis la presse islamique, les musulmans francophones ont développé la problématique de la laïcité dans des formats peu habituels.

\footnotetext{
${ }^{12}$ Loi $n^{\circ}$ 019-2000/AN du 27 juin 2000.

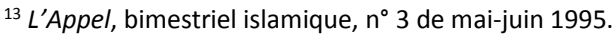

${ }^{14}$ Al-Maidane, bimensuel, $n^{\circ} 22$ du $1^{\text {er }}$ au 15 mars 2000, p. 3.
} 


\section{Un colloque sur la question de la laïcité}

L'élite francophone musulmane en puisant dans son propre répertoire culturel occidental a initié depuis les années 1990 des rencontres novatrices qui invitent au débat. Sous forme de conférences, de causeries ou encore de dîners-débats, ces cadres réunissent le plus souvent des intellectuels autour de sujets précis. La thématique de la laïcité a fait l'objet d'un colloque organisé en août 2007 par le CERFI à Ouagadougou : "Quelle laïcité pour une société plurielle et prospère ? ". La neutralité étatique, l'identité musulmane (foulard), l'éducation et le trafic d'influence administrative à des fins de prosélytisme furent les principaux axes du colloque. Les communications et les échanges ont attesté de façon globale l'existence d'une pratique laïque qui favorise le christianisme au détriment de l'islam. Il a été mentionné par exemple l'existence de cercles de chrétiens dans les services qui mèneraient une politique d'isolement des musulmans, la fixation des congés scolaires et universitaires sur la base du calendrier des fêtes chrétiennes, l'identité musulmane des filles bafouée dans les écoles, le déséquilibre constaté dans la répartition des plages horaires accordées aux confessions religieuses à la RadiodiffusionTélévision du Burkina, les subventions de 500 millions accordés à l'enseignement chrétien (Traoré, 2010 : 45-46).

Il est clair qu'une certaine mobilisation musulmane s'est faite autour de cette question de la laïcité. Même si les groupes musulmans sensibilisés sont minoritaires dans la mesure où le débat est circonscrit autour du cercle des musulmans francophones, le problème de la gestion de la laïcité est identifié, connu, médiatisé et débattu. Avec la tenue du colloque, il est évident que les communications ont permis à cette élite intellectuelle de préciser sa perception de la laïcité et éventuellement d'affiner ses stratégies. La création d'un observatoire de la laïcité fut l'une des recommandations formulées par le colloque (Traoré, 2010 : 50).

Les différentes dynamiques que nous analysons et qui concourent à la publicisation des problèmes ne sont ni isolées ni inscrites dans un ordre chronologique. Concrètement, ces dynamiques de mobilisation collective, de médiatisation et de politisation s'entrelacent et s'imbriquent. Ce problème de la gouvernance de la laïcité inscrit dans l'agenda de certains 
groupes musulmans va passer par un concours de circonstances à l'agenda public.

Le problème de la gouvernance de la laïcité, de l'agenda musulman à l'agenda public

\section{La publicisation du problème de la gouvernance de la laïcité}

Le Burkina Faso a été secoué en avril 2011 par une crise sociopolitique née d'une mutinerie au sein du Régiment de sécurité présidentielle communément appelé RSP. Cette crise est partie d'un mouvement de protestation né à la suite de la mort d'un élève interpellé par la police dans la ville de Koudougou, pour s'étendre ensuite à des garnisons militaires. Elle s'est traduite par des scènes de violences meurtrières, de pillages et de destruction de biens (Hilgers et Loada, 2013). Dans la perspective d'une résolution de la crise, le président du Burkina Faso, Blaise Compaoré, initie un dialogue ouvert avec les différentes composantes sociopolitiques ainsi que les autorités coutumières et religieuses. II met en application un projet, sous le format de réformes politiques qu'il avait annoncées le 11 décembre 2009 dans son discours à la nation prononcé à l'occasion de la célébration de la fête nationale. Cette idée de réformes politiques avait été également inscrite dans le programme quinquennal 2010-2015 que le président a défendu lors des campagnes électorales présidentielles. L'objectif affiché était l'approfondissement de la démocratie. La crise sociopolitique de 2011 en accélère le processus. Ce cadre de réflexion prend le nom de Conseil consultatif sur les réformes politiques (CCRP) créé par le décret du 10 mai 2011. Il était composé de 68 membres issus des partis politiques, de la société civile et des confessions religieuses et coutumières. Les travaux ont porté sur quatre axes à savoir l'extension du dialogue démocratique par la création de nouveaux cadres d'expression, la redéfinition des rapports entre les pouvoirs exécutif, législatif et judiciaire, l'amélioration de la gouvernance électorale et le renforcement de la gouvernance globale. Ce n'est pas la première fois que les confessions religieuses et coutumières sont impliquées ou associées à la résolution des crises ou à l'entreprise d'actions publiques. Depuis la reprise du processus démocratique en 1991 par exemple, elles ont participé activement à la formation du Collège des sages en 1999, à la célébration de la Journée 
nationale du pardon en 2001 et aux activités de la Commission nationale d'organisation des élections (CENI).

Au sein de ce CCRP, les trois confessions religieuses (musulmane, catholique et protestante) furent représentées chacune par trois délégués ${ }^{15}$. Le Conseil a eu pour mission du 23 juin au 14 juillet 2011 d'examiner les réformes proposées par les acteurs de la vie nationale. Cette opportunité a été saisie par les conseillers musulmans mandatés par la Fédération des associations islamiques du Burkina (FAIB), pour plaider la mise en place d'un observatoire de la laïcité, sorte d'organe de veille. Créée en 2005 , la FAIB, structure faîtière des associations islamiques, a en effet été un cadre propice à l'élite intellectuelle francophone pour faire entendre ses préoccupations, dont celle portant sur la laïcité ${ }^{16}$. Le motif avancé par les conseillers musulmans est l'existence d'un traitement inégalitaire des différentes religions par des structures et agents de l'État. Il faut rappeler que l'islam burkinabè historiquement subordonné au pouvoir politique depuis le temps précolonial (Otayek, 1984: 300) n'a jamais développé un mouvement contestataire mais, dans l'imaginaire populaire musulman, les fidèles de l'islam sont défavorisés par rapport aux chrétiens (Otayek, 1984: 313 ; Samson, 2013: 185-189). II n'est pas étonnant que l'islam burkinabè à travers ses élites s'inscrive dans une logique de lutte pour plus de reconnaissance dans l'espace public (Samson, 2013 : 189 ; Holder et Saint-Lary, 2014). Après des discussions au sein du CCRP, un consensus ne s'est pas dégagé pour la création dudit observatoire. Les conseillers membres du CCRP ont soutenu en majorité que le principe de la laïcité n'est pas remis en cause et qu'il n'existe pas de problèmes religieux majeurs. L'hypothèse d'une tentative de déni d'agenda est soutenable dans la mesure où des groupes et individus se sont prononcés défavorablement à l'idée de création d'un observatoire. Selon I'approche de Cobb et Ross (1997), cités par Kubler et De Maillard

${ }^{15}$ Catholiques : Ignace Sandwidi, Rosine Booro, Marc Somda. Protestants : pasteur Henri Yé, pasteur Jean Charles Kabré, Mathias Niambekoudougou. Musulmans: El Hadj Adama Sakandé, El Hadj Souleymane Compaoré, Youssouphe Kientega.

${ }^{16}$ En 2008, 198 associations islamiques étaient membres de la FAIB. On y trouve plusieurs courants dont les confréries, les réformistes wahhabites, les réformistes francophones, etc. L'AEEMB et le CERFI occupent au sein de la FAIB deux des huit postes de vice-présidence. 
(2009), il s'agit ici d'une stratégie de déni d'agenda par non-reconnaissance du problème. Toutefois, un consensus se dégage en faveur de l'organisation d'un débat national sur la laïcité traduit en recommandation ${ }^{17}$. Pour contrer cette stratégie de déni d'agenda et montrer la pertinence de la mise à l'agenda du problème de la laïcité, le secrétaire de la FAIB, Souleymane Compaoré, publie un écrit après les assises du CCRP où il revient sur les statistiques démographiques et sur l'iniquité constatée dans la représentation des confessions religieuses au sein des organes et instances. L'exemple pris porte même sur le cas de la représentativité musulmane au CCRP :

"Cela pour faire remarquer que les musulmans, 60,5\% de la population selon les chiffres officiels... étaient représentés par trois (3) délégués alors que les chrétiens (catholiques et protestants réunis), moins de $24 \%$ de la population toujours selon les données officielles, avaient six (6) représentants au CCRP18 ".

Avec les différentes plaintes et la mobilisation médiatique qui a suivi, les mobiles pour poser officiellement la problématique de la laïcité étaient réunis. La crise sociopolitique de 2011 donna l'opportunité à la FAIB de porter le débat dans l'espace public. Elle mobilisa plusieurs ressources dont l'unité musulmane, la politisation des statistiques démographiques et la manipulation des quotas représentatifs appliqués pour convaincre les autres acteurs de la pertinence du problème.

Les acteurs du forum national: conflits, négociations et compromis

Les travaux du CCRP ont été suivis par l'organisation des assises nationales du 7 au 9 décembre 2011 sur les réformes politiques. Ces assises nationales ont regroupé 1510 personnes en provenance des 13 régions du pays et elles étaient chargées d'examiner et d'adopter les propositions faites par le CCRP. Elles ont débouché dans leurs travaux sur des propositions consensuelles de réformes soumises au gouvernement : l'élaboration du

\footnotetext{
${ }^{17}$ Rapport général de la session du Conseil consultatif sur les réformes politiques (CCRP), tenue du 23 juin au 14 juillet 2011, Ouagadougou, 128 p.

18 Écrit de Souleymane Compaoré, secrétaire général de la Fédération des associations islamiques du Burkina (FAIB), paru dans le quotidien Sidwaya, du 19 juillet 2011.
} 
statut de la chefferie traditionnelle, l'organisation d'un débat national sur la laïcité, la constitutionnalisation du genre, la création d'un sénat, la constitutionnalisation du médiateur du Faso, I'amnistie accordée aux anciens chefs de l'État, etc.

Sur la question de l'organisation du débat national sur la laïcité, le gouvernement a décidé de tenir un forum, un cadre propice aux discussions. L'objectif était de réfléchir sur la compréhension partagée du concept de laïcité afin de renforcer sa pratique comme facteur de paix et de cohésion sociale, d'identifier les attitudes négatives et de proposer des recommandations et des mesures appropriées ${ }^{19}$. Mais avant la tenue effective dudit forum, la partie musulmane a exigé une application équitable du quota des représentants des confessions religieuses, c'est-àdire la parité dans la répartition musulmans/chrétiens ${ }^{20}$. Cette doléance de la faîtière des associations musulmanes qui s'apparente à une forme de pression a reçu un écho favorable. La FAIB fut représentée par 20 délégués ${ }^{21}$, la Conférence épiscopale Burkina-Niger par 10 délégués et la Fédération des églises et missions évangéliques par 10 délégués. Un responsable d'association islamique délégué au forum trouvait la répartition classique non équitable et la discutait en ces termes :

"On sépare les catholiques à part et les protestants à part. Si on va rentrer dans cette logique, les musulmans peuvent présenter au

\footnotetext{
${ }^{19}$ Rapport général du forum national sur la laïcité au Burkina Faso, 27-29 septembre 2012, Ouagadougou, $110 \mathrm{p}$.

20 Le quota des représentants se faisait à part égale entre musulmans, catholiques et protestants. La conséquence était que le nombre de représentants chrétiens (catholiques et protestants) fut le double de celui des musulmans.

21 Les 20 représentants musulmans étaient composés de francophones et de francoarabisants. Parmi les francophones, on peut citer Souleymane Compaoré (journaliste), Ismael Tiendrebeogo (juriste), Nouhoun Bagayoko (géographe), Maimouna Kone (professeur), Harouna Sana (policier à la retraite), Oumarou Ganamé (arabisant), Aboubacar Yugo (arabisant), Maïga Aboubacari, Hadja Traoré Aïssata, Bambara Amadé, Ouédraogo Séni, Ouédraogo Abdoulaye (professeur), Sawadogo Louqman, Sawadogo Kadré, Soumano Boubacar, Ouédraogo Mariam, Semdé Issaka, Diakité Sériba, Sawadogo Moussa, Bancé Ousmane.
} 
moins quatre groupes. Nous avons souhaité que le quota soit équitable entre musulmans et chrétiens ${ }^{22}$ ".

En analysant les débats menés au forum, il se dégage deux grandes perceptions de la laïcité. Dans une première attitude, les différents acteurs qu'ils soient de l'État, de la société civile ou des confessions religieuses ont soutenu des principes tels que l'autonomie des sphères étatique et religieuse, la non-ingérence réciproque, la neutralité idéologique de l'État, la bienveillance de l'État à l'égard des religions (non au laïcisme). II s'agit d'une tradition de collaboration entre l'État et les confessions religieuses fortement encouragée ${ }^{23}$. Comme au Sénégal, les confessions religieuses du Burkina Faso plaident en faveur d'une "laïcité de collaboration" (De Benoist, 2003 ; Dione, 2013 : 111) et s'inscrivent donc dans ces «laïcités à l'africaine " où les religions sont associées au fonctionnement des États sans qu'elles prétendent se substituer à ces États (Kaag et Saint-Lary, 2011).

La deuxième posture révèle des perceptions plus nuancées dans la mesure où il n'existe pas de définition juridique de la laïcité. Les représentations livrées par les acteurs à propos de ce concept sont dictées par leurs intérêts spécifiques. Selon J. Bauberot (2013: 11), un acteur peut être amené à se faire une représentation de la laïcité qui privilégie un principe au détriment des autres. Comme il fallait $s^{\prime} y$ attendre, la partie gouvernementale, relayée par le Premier ministre, le ministre chargé des Réformes politiques, le ministre de l'Administration territoriale, a dépeint un contexte mondial (caricatures provocatrices de l'islam, islamisme au Mali et Nigeria) inquiétant. D'où la focalisation de ses interventions autour des questions de paix, sécurité, cohabitation, tolérance, prévention de dérives extrémistes et démocratie comme cadre propice à

${ }^{22}$ Adama Sakandé, vice-président et président par intérim de la CMBF, entretien du 17 janvier 2013 à Ouagadougou.

${ }^{23}$ Les autorités religieuses et coutumières figurent dans le protocole d'État, l'État est impliqué dans l'organisation du pèlerinage à La Mecque à travers la création d'une commission nationale d'organisation, les leaders religieux et coutumiers sont sollicités par l'État lors des crises sociopolitiques, les confessions religieuses participent à l'organisation des élections, etc. 
l'épanouissement de la laïcité ${ }^{24}$. La chefferie traditionnelle estime qu'elle a fait montre d'un esprit de laïcité en accordant une hospitalité aux religions révélées que sont l'islam et le christianisme. Elle recommande par conséquent la préservation de la cohésion sociale et de la cohabitation religieuse entretenues depuis longtemps. La partie catholique, en corrélation avec ce même contexte mondial, s'est préoccupée plus de sa survie en insistant sur les questions de liberté de conscience et de croyance, de dialogue, de tolérance et de violence religieuse. II faut rappeler que, depuis les années 1970, la hiérarchie catholique s'est inquiétée des progrès de l'islam tant du point de vue démographique que de sa présence dans l'espace public. Elle a même craint une islamisation progressive des institutions de l'État à travers l'augmentation du nombre de musulmans membres du gouvernement, le projet d'adhésion du pays à l'Organisation de la conférence islamique $(\mathrm{OCI})$, l'émission de timbres d'inspiration musulmane (Audouin et Deniel, 1975 : 70). Cette inquiétude pourrait se renforcer avec le poids démographique croissant des musulmans et la montée d'une société civile musulmane plus revendicatrice. Quant à la partie protestante, elle a été plus critique envers l'État qui prendrait des colorations islamiques avec son implication dans l'organisation du hadj et un projet de création d'un ministère chargé des Affaires islamiques ${ }^{25}$. En insistant sur la protection de la liberté de conscience et de religion, le respect de la spécificité religieuse, elle s'inquiète des menaces islamistes qui pèsent sur le Burkina Faso à cause de la présence d'Ansar Dine à Bobo-Dioulasso ${ }^{26}$ : « Les effets sont déjà à nos portes avec la dislocation du Mali » avance la partie protestante. L'argumentaire présenté par les musulmans n'est qu'une reprise de ce qu'ils avaient déjà construit autour de la laïcité. La laïcité " à la française » est rejetée comme modèle inadapté au cas burkinabè. Les délégués

${ }^{24}$ Rapport général du forum national sur la laïcité au Burkina Faso, 27-29 septembre 2012, Ouagadougou, $110 \mathrm{p}$.

${ }^{25}$ L'existence d'un tel projet n'a pas pu être vérifiée sur le terrain.

${ }^{26}$ II s'agit d'une confusion entre Ansar Dine, mouvement islamiste au Mali qui défraie la chronique en 2012, et une autre association portant le même nom. Celui de Bobo-Dioulasso est une section du mouvement soufi créé par Cheikh Haidara au Mali et connu pour son caractère non violent. 
musulmans émettent un doute quant à la mise en œuvre pratique de la laïcité par l'État en rappelant la présence de l'esprit discriminatoire :

"Ce constat est quelquefois récusé avec mauvaise foi au motif qu'aucune politique officielle de l'État burkinabè ne fonde une discrimination à l'égard des autres communautés religieuses. Si cet argument est fondé en droit, certaines pratiques de l'État corrompent le droit positif ${ }^{27}$ \%.

La FAIB a fait du traitement égalitaire des confessions religieuses son cheval de bataille et s'est prononcée en faveur d'une "laïcité positive ". Dans cette laïcité, l'État soutient les confessions religieuses et tient compte de l'équité dans leur traitement :

"Si dans la démocratie, toute majorité ne peut pas faire la loi surtout quand il existe des critères de majorité qualifiée, il est anachronique que les groupes minoritaires soient mieux représentés que les groupes majoritaires... Si l'injustice faite à l'islam, relativement à sa sous-représentation dans les instances et organes créés par l'État, semble avoir trouvé un début de solution avec ce forum, nous souhaitons de tous nos voux que le droit soit définitivement aligné sur le fait...28 ${ }^{2}$.

Les mêmes dispositions sont réclamées pour résoudre certains problèmes comme le port du voile :

"De même, les pouvoirs publics devraient rappeler une fois pour toute qu'aucune loi n'interdit le port du voile au Burkina Faso; et par conséquent prendre des mesures afin que cessent les différentes tracasseries constatées... discrimination à l'embauche, interdiction d'accès à des salles de classe ou de concours alors que l'identification de la personne est possible ${ }^{29}{ }^{\prime}$.

Les échanges entre les différents acteurs ont donné droit à des compromis et à des concessions sous forme de recommandations. Ainsi, les musulmans acceptèrent le maintien de la trilogie spécifique "musulmans, catholiques, protestants" tandis que les catholiques et

\footnotetext{
${ }^{27}$ Rapport général du forum national sur la laïcité au Burkina Faso, 27-29 septembre 2012, Ouagadougou.

${ }^{28} \mathrm{lbid}$.

${ }^{29}$ lbid.
} 
protestants se conformèrent au respect de l'équité entre christianisme et islam au niveau du nombre de délégués dans les organes représentatifs. Dans une perspective de mise en place d'une feuille de route, les membres du forum ont recommandé l'institutionnalisation du forum sur la laïcité, la formation à la laïcité, la culture du dialogue interreligieux et l'élaboration d'un document référentiel sur la laïcité.

Certes, la publicisation du problème de la laïcité fut une coproduction de plusieurs acteurs, mais l'État en a été le maillon essentiel. C'est l'État qui a décidé du cadrage et de la suite à donner à la mise à l'agenda. Cependant, on est en droit de s'interroger sur le sens de la démarche menée par l'État burkinabè. Répond-elle à un souci de recherche de légitimité dans un contexte d'affaiblissement du pouvoir en place ou répond-elle plutôt à une véritable volonté d'associer la société civile et religieuse à l'action publique ?

Adama Fofana ${ }^{30}$, dans un article de presse, assimile l'attitude de l'État à une fuite de responsabilité synonyme de faiblesse. Pour lui, l'État et les cultes n'ont pas à décider ensemble de ce que doit être la laïcité. C'est une prérogative qui relève de l'État habilité à légiférer sur la question pour plus de neutralité et d'équité. Voilà pourquoi il fait une analyse critique et soutient que les préoccupations soulevées sur la question de la laïcité devraient préalablement être traitées par les pouvoirs exécutif et législatif afin d'éviter des risques de vacuité ${ }^{31}$. Au-delà d'une éventuelle faiblesse de l'État, l'attitude du gouvernement pourrait s'inscrire aussi dans le cas de figure où l'État, dans une perspective démocratique, ne décide pas seul, mais s'engage à jouer le rôle de régulateur de concert avec les autres acteurs. Cela dénote une prise en compte sérieuse de la question, et la création par le gouvernement en janvier 2015 d'un Observatoire national des faits religieux (ONAFAR) milite en faveur de cette position. Organe

\footnotetext{
${ }^{30}$ Ancien ministre chargé des relations avec le Parlement, porte-parole du gouvernement. II est le président de La Petite Académie, un site web lancé en novembre 2004 pour promouvoir le patrimoine historique du Burkina Faso.

31 Fofana Adama, "Éviter les risques de vacuité ", journal quotidien Le Pays, nº 5201 du 25 septembre 2012.
} 
consultatif constitué de dix membres ${ }^{32}$, il est chargé entre autres de surveiller les contenus médiatiques à caractère religieux, de veiller à l'application de la règlementation sur les pratiques cultuelles, et de promouvoir la tolérance et le dialogue interreligieux.

\section{Conclusion}

La tenue d'un débat public sur la question de la laïcité au Burkina Faso montre un exemple de coproduction d'action publique. La frange des musulmans francophones défend une sorte de laïcité endogène, plaide en faveur d'un traitement égalitaire des confessions religieuses et propose la création d'un observatoire. Pour y parvenir, ces élites musulmanes occidentalisées ont usé de stratégies comparatistes et de ressources symboliques comme les statistiques stratégiquement exploitées. II y a eu des tentatives de déni d'agenda, mais finalement les acteurs s'accordent pour la tenue d'un forum en lieu et place de la mise en place d'un observatoire. Si les plaintes musulmanes ont eu un impact dans la publicisation du problème, il ne faut pas écarter les conjonctures qui ont favorisé cette mise à l'agenda. Face au spectre de l'islamisme qui se développe dans le monde et également dans la sous-région, ce débat sur la laïcité se présentait finalement comme une opportunité pour les acteurs, l'État en premier lieu. Lors du forum, les enjeux n'étaient pas les mêmes: prisme sécuritaire pour l'État, traitement égalitaire pour les musulmans, et crainte d'une remise en cause de la liberté religieuse pour les catholiques et protestants. En fin de compte, par l'interaction de plusieurs parties, le problème posé par les musulmans francophones est formulé, discuté, reformulé, redimensionné conformément aux enjeux locaux et globaux (sécuritaire, menace islamiste).

Au-delà du cas burkinabè, la question du traitement égalitaire des différentes confessions religieuses réclamé par les communautés musulmanes se pose dans les pays d'Afrique subsaharienne. Elle

\footnotetext{
${ }^{32}$ Mandatés par le Conseil supérieur de la communication, du ministère de l'Administration territoriale, la Fédération des associations islamiques du Burkina, la Fédération des églises et missions évangéliques et la Conférence épiscopale Burkina-Niger.
} 
s'interprète également comme les signes de l'émergence d'une société civile musulmane qui, sans être contestatrice, frondeuse ou rebelle, s'inscrit dans un courant critique dans les rapports entre l'État et les confessions religieuses. Un projet de loi sur les libertés religieuses initié par le gouvernement burkinabè et retiré en janvier 2016 sous la pression de la Fédération des associations islamiques du Burkina confirme davantage l'émergence de cette société civile. La FAIB a estimé que certaines dispositions de la loi réduisent la liberté de conscience et de culte et qu'il était nécessaire de procéder à une large concertation afin d'aboutir à des propositions consensuelles.

\section{Bibliographie}

AUDOUIN J. et DENIEL R., 1975, L'islam en Haute-Volta à l'époque coloniale, Abidjan, Inades.

BAUBEROT J., 2013, "Sécularisation, laïcité, laïcisation ", in HOLDER G. et SOW M. (éds), L'Afrique des laïcités. État, religion et pouvoirs au sud du Sahara, Bamako, IRD et Éditions Tombouctou : 5-18.

BENOIST J.R. de, 2003, "Laïcité et laïcisme au Sénégal au xxe siècle » in DELISLE P. et SPINDLER M. (éds), Les relations Églises-État en situation postcoloniale. Amérique, Afrique, Asie, Océanie XIXe-xxe siècles, Paris, Karthala : 231-248.

BOURON J.M., 2010, « Les pères blancs, acteurs du jeu colonial. Mission catholique et enjeux politiques en Haute-Volta au lendemain de la seconde guerre mondiale ", Histoire, monde et cultures religieuses, 14(2) : 59-81.

CIMEF, 2001, Les musulmans francophones, réflexions sur la compréhension, la terminologie, le discours, Paris, Éditions Tawhid.

COBB R. et ROSS M., 1997, "Agenda setting and the denial of agenda access: key concepts ", in COBB R. et ROSS M. (eds.), Cultural strategies of Agenda Denial: Avoidance, Attack and Redefinition, Lawrence, University Press of Kansas : 3-23.

DENIEL R., 1970, Croyances religieuses et vie quotidienne. Islam et christianisme à Ouagadougou, collection Recherches voltaïques, $n^{\circ} 14$, ParisOuagadougou, CNRS-CVRS. 
DIONE M.S., 2013, “ De la réception-réappropriation du concept de laïcité au Sénégal, ou de la spécificité d'une laïcité de collaboration entre le politique et le religieux ", in HOLDER G. et SOW M. (éds), L'Afrique des laïcités. État, religion et pouvoirs au sud du Sahara, Bamako, IRD et éditions Tombouctou : 111-124.

GUY N., 1981, Dynamique de l'islam au Sud du Sahara, Paris, ALC.

HILGERS M. et LOADA A., 2013, «Tensions et protestations dans un régime semiautoritaire : croissance des révoltes populaires et maintien du pouvoir au Burkina Faso », Politique africaine, 131 : 187-208.

HOLDER G. et SAINT-LARY M., 2014, « Enjeux démocratiques et (re)conquête du politique en Afrique. De l'espace public religieux à l'émergence d'une sphère islamique oppositionnelle ", Sens public: 2-17, http://senspublic.org/article1086.html (consulté le 31 mars 2019).

HOLDER G. et SOW M., 2013, " Les laïcités africaines vues de Bamako : un colloque pris par son contexte ", in HOLDER G. et SOW M. (éds), L'Afrique des laïcités. État, religion et pouvoirs au sud du Sahara, Bamako, IRD et éditions Tombouctou : 19-37.

KAAG M. et SAINT-LARY M., 2011, "Nouvelles visibilités de la religion dans l'arène du développement ", Bulletin de l'APAD, 33 : 1-11.

KUBLER D. et MAILLARD J. de, 2009, Analyser les politiques publiques, Paris, Presses universitaires de Grenoble.

MIRAN-GUYON M., 2013, "Gloires et déboires de la laïcité en Côte d'ivoire au prisme de l'imaginaire musulman ", in HOLDER G. et SOW M. (éds), L'Afrique des laïcités. État, religion et pouvoirs au sud du Sahara, Bamako, IRD et éditions Tombouctou : 315-328.

OTAYEK R., 1984, « La crise de la communauté musulmane de Haute-Volta : I'islam voltaïque entre réformisme et tradition, autonomie et subordination » Cahiers d'études africaines, 95 : 299-320.

OTAYEK R., 1997, "L'Église catholique au Burkina Faso. Un contre-pouvoir à contretemps de l'histoire ?", in CONSTANTIN F. et COULON C. (éds), Religion et transition démocratique en Afrique noire, Paris, Karthala : 221258.

OUÉDRAOGO Y., 2013, " Diversité musulmane et laïcité au Burkina Faso », Cahiers du CERLESHS, 45 : 1-28. 
SAMSON F., 2013, "La concurrence islamique comme enjeu de laïcité de l'État. Exemples comparés du Sénégal et du Burkina Faso ", in HOLDER G. et SOW M. (éds), L'Afrique des laïcités. État, religion et pouvoirs au sud du Sahara, Bamako, IRD et éditions Tombouctou : 182-191.

TRAORÉ B., 2010, "À la recherche d'une voie africaine de la laïcité. Islam et pluralisme religieux au Burkina Faso ", Islam et sociétés au sud du Sahara, Diversité et habits singuliers, $\mathrm{n}^{\circ} 2$, Paris, Les Indes savantes : 9-54.

VITALE M., 2013, "Quelle laïcité au Burkina ? Les enjeux du dialogue entre État, religions et citoyens ", in HOLDER G. et SOW M. (éds), L'Afrique des laïcités. État, religion et pouvoirs au sud du Sahara, Bamako, IRD et éditions Tombouctou : 303-311.

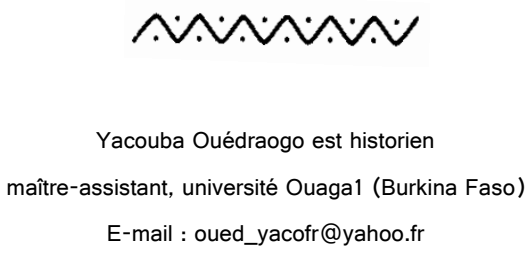

